\title{
Entwicklung eines Hochtemperaturmessgerätes für die elektrische Leitfähigkeit, die Hall-Konstante und den Seebeck-Koeffizienten
}

\author{
$\underline{\text { Robin Werner }}$, Jaroslaw Kita ${ }^{1}$, Michael Gollner ${ }^{2}$, Florian Linseis ${ }^{2}$ und Ralf Moos ${ }^{2}$ \\ ${ }^{1}$ Lehrstuhl für Funktionsmaterialien, Universität Bayreuth, Bayreuth, Deutschland \\ 2Linseis Messgeräte GmbH, Selb, Deutschland \\ Kontakt: Functional.Materials@Uni-Bayreuth.de
}

\section{Einleitung}

Die elektrische Leitfähigkeit, die Hall-Konstante und der Seebeck-Koeffizient beschreiben maßgeblich die wichtigsten elektrischen Transporteigenschaften von Materialien. Die Messung der o.g. temperaturabhängigen Parameter erfordert dabei mehrere, oft aufwändige Messgeräte. Ein Blick auf die kommerziell verfügbaren Messgeräte verrät, dass die elektrische Leitfähigkeit entweder zusammen mit der Hall-Konstante oder mit dem Seebeck-Koeffizienten gemessen wird.

Bei den Geräten bzw. Aufbauten zur Messung des Seebeck-Koeffizienten wird dazu oft ein Ofen auf die gewünschte Messtemperatur geregelt. Ein weiterer, in dem Ofen verbauter Heizer sorgt für einen Temperaturgradienten innerhalb der Probe und ermöglicht die Messung einer Thermospannung und daraus die Bestimmung des Seebeck-Koeffizienten.

Für die Messungen der Hall-Konstante hingegen werden eine homogene Temperaturverteilung und ein zusätzliches Magnetfeld benötigt. Das Magnetfeld kann entweder über Permanentmagneten oder über Elektromagneten erzeugt werden. Wichtig dabei ist die Ausrichtung der magnetischen Feldlinien, welche senkrecht zur Richtung des durch die Probe des fließenden Stromes angeordnet sein sollten. Die magnetische Flussdichte sollte dabei unabhängig von der Erzeugungsart so groß wie möglich sein, um die

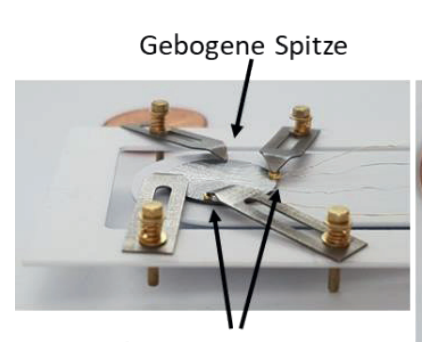

$\mathrm{Au} / \mathrm{Pt}$-Thermoelemente

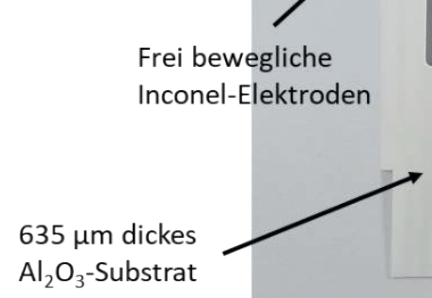

Probenfläche $\min . \varnothing 5 \mathrm{~mm}$

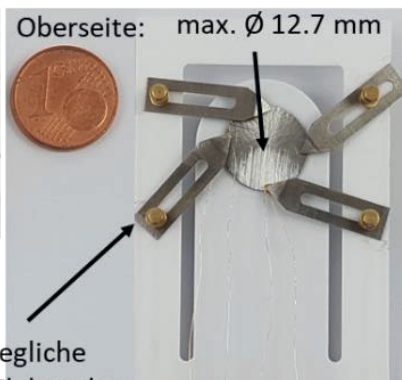
$\mathrm{Al}_{2} \mathrm{O}_{3}$-Substrat
Messgenauigkeit der Hall-Spannung zu erhöhen. Dabei spielen der Polabstand und die Polflächen eine wichtige Rolle. Über den Polabstand lässt sich die Stärke der Flussdichte eines Magnetfeldes einstellen, während die Größe der Polflächen primär die Homogenität bestimmt. Um bei einer betragsmäßig hohen magnetischen Flussdichte messen zu können, ist daher ein möglichst niedriger Polabstand zu wählen. Der geringe Abstand macht die Verwendung von sehr kleinen, nicht-magnetischen Öfen oftmals kompliziert und erschwert damit eine Integration der Messung des Seebeck-Koeffizienten.

Im Rahmen dieses Beitrages wird über den Stand der Entwicklung eines Hochtemperatur-Kombimessgerätes zur Messung der elektrischen Leitfähigkeit, der Hall-Konstante und des Seebeck-Koeffizienten berichtet. Dabei wird speziell auf die Erweiterung eines zuvor in Dickschichttechnologie entwickelten Hochtemperatur-Hall-Messplatzes um die Funktion der Seebeck-Messungen und deren Herausforderungen eingegangen [1].

\section{Probenträger in Dickschichttechnologie}

Der neue Probenträger besteht aus einem $\mathrm{Al}_{2} \mathrm{O}_{3}$-Substrat auf dessen Unterseite abgedeckte siebgedruckte Heizleiterstrukturen aufgebracht wurden (Abb. 1). Durch Joule'sche Erwärmung der primären Heizleiterstruktur kann der Probenträger und somit

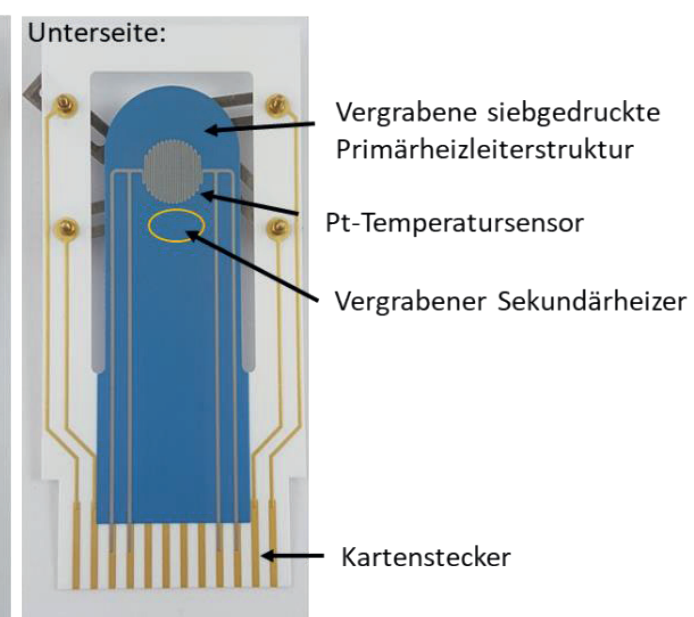

Abb. 1: Probenträger aus Aluminiumoxid mit siebgedruckten Heizleiterstrukturen, frei beweglichen Elektroden, zwei Au/Pt-Thermoelementen für Messungen der elektrischen Leitfähigkeit, der Hall-Konstanten und des Seebeck-Koeffizienten bis $800^{\circ} \mathrm{C}$ 
auch eine auf der Oberseite platzierte Probe bis zu einer Temperatur von $800{ }^{\circ} \mathrm{C}$ erhitzt werden. Ein zusätzlicher Sekundärheizer erlaubt das Einstellen einer variablen Temperaturdifferenz innerhalb der Probe für Messungen des Seebeck-Koeffizienten.

Auf der Oberseite befinden sich vier freibewegliche Inconel-Elektroden, zwischen denen Proben beliebiger Geometrie bis zu einem Durchmesser von 12,7 mm nach der Van-der-Pauw-Messmethode kontaktiert werden können [2]. Die für die Bestimmung des Seebeck-Koeffizienten erforderliche Kontaktstellentemperatur kann über die Thermospannungen zweier Au/Pt-Thermoelemente gemessen werden, die zwischen Elektrode und Probe geklemmt werden [3].

Die Funktionsfähigkeit dieses Probenträgerkonzeptes in Dickschichttechnologie für die einzelnen elektrischen Transportparameter konnte bereits in vorangegangenen Arbeiten gezeigt werden [1,4].

\section{Herausforderungen}

In diesem Beitrag wird auf die Herausforderungen eingegangen, die die Messung der elektrischen Leitfähigkeit, der Hall-Konstante und des Seebeck-Koeffizienten mit den aus Abb. 1 dargestellten Probenträger in einem Messzyklus mit sich bringen. Ein besonders wichtiger Punkt ist dabei der sogenannte „ColdFinger-Effekt".

Über die Thermoelemente/Inconel-Elektroden wird ein Wärmestrom aus der Probe nach außen in den kalten Bereich abgeführt. Dadurch reduziert sich an den elektrischen Kontaktstellen die Temperatur (siehe Abb. 2).

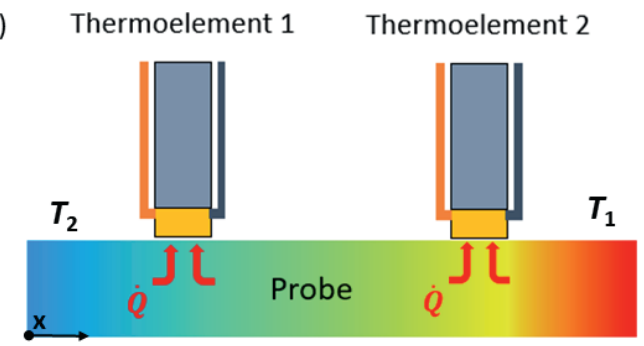

b)

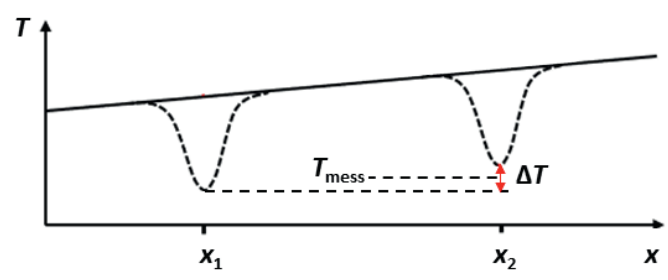

Abb. 2: Schematische Darstellung des Cold-Finger-Effekts a) Temperaturverlauf zwischen zwei Thermoelementen während der Messung des Seebeck-Koeffizienten b) Temperaturprofil mit angedeutetem Cold-Finger-Effekt an den elektrischen Kontakten
Für die Messungen des Seebeck-Koeffizienten ist die Kontaktstellentemperatur allerdings besonders wichtig, da zwischen den Thermoelementen nicht nur die Temperaturdifferenz gebildet, sondern aus deren Mittelwert auch die Messtemperatur des SeebeckKoeffizienten bestimmt wird (Abb. 2b). Ist dieser Cold-Finger-Effekt besonders stark ausgeprägt, kann es vorkommen, dass die Messtemperatur des Seebeck-Koeffizienten deutlich unterhalb der Messtemperatur der Van-der-Pauw-Messungen liegt, was sowohl Messfehler als auch eine eingeschränkte Vergleichbarkeit der Messergebnisse nach sich zieht $[5,6]$. Mit Hilfe von FE-Simulationen konnte dieser Effekt signifikant verringert und dadurch der Messaufbau wesentlich verbessert werden.

Im Rahmen dieses Beitrages soll mithilfe von Messungen und FE-Simulationen auf den Einfluss der Thermoelementgeometrie auf den Cold-Finger-Effekt und die daraus resultierenden Vor- und Nachteile für ein solches Hochtemperatur-Kombimessgerät eingegangen werden.

\section{Literatur}

[1] WERNER, R.; KITA, J.; GOLLNER, M.; LINSEIS, F.; MOOS, R.: Novel, low-cost device to simultaneously measure the electrical conductivity and the Hall coefficient from room temperature up to $600{ }^{\circ} \mathrm{C}$, J. Sens. Sens. Syst. 10 (2021), S. 71-81, doi: 10.5194/jsss-1071-2021

[2] VAN DER PAUW, L.J.: A Method of Measuring Specific Resistivity and Hall Effect of Discs of Arbitrary Shape, In: Semiconductor Devices: Pioneering Papers, edited by: SZE, S. M., World Scientific, Singapore, S. 174-182, https://doi.org/10.1142/9789814503464_0017, 1991

[3] KIM, Y.-G.; GAM, K.S.; KANG, K.H.: Thermoelectric properties of the Au/Pt thermocouple, Sci. Instrum. 69 (1998), S. 3577-3582, doi: 10.1063/1.1149141

[4] WERNER, R.; KITA, J.; GOLLNER, M.; LINSEIS, F.; MOOS, R.: Adding Seebeck coefficient measurements to an existing high temperature device for Hall constant and electrical conductivity measurements, SMSI Sensor and Measurement Science International, Virtual Conference, 03-06 May 2021, Nuremberg, Germany, doi: 10.5162/SMSI2021/A6.2

[5] BERNHARD, F.: Handbuch der Technischen Temperaturmessung, 2. Edition, Springer, Berlin, Heidelberg, ISBN 978-3-642-24505-3, 2014

[6] BORUP, K.; DE BOOR, J.; WANG, H.; DRYMIOTIS, F.; GASCOIN, F.; SHI, X.; CHEN, L.; FEDOROV, M.; MÜLLER, E.; IVERSEN, B.; SNYDER, G.J.: Measuring thermoelectric transport proper-ties of materials, Energy Environ. Sci. 8 (2015), S. 423-435, doi: 10.1039/c4ee01320d 